\title{
Feasibility of dual-low scheme combined with iterative reconstruction technique in acute cerebral infarction volume CT whole brain perfusion imaging
}

\author{
TAO WANG $^{1}$, YI GONG ${ }^{2}$, YIBING SHI $^{1}$, RONG HUA $^{1}$ and QINGSHAN ZHANG $^{3}$ \\ ${ }^{1}$ CT Room, Departments of ${ }^{2}$ Ultrasound and ${ }^{3}$ Neurology, Xuzhou Central Hospital, Xuzhou, Jiangsu 221009, P.R. China
}

Received August 15, 2016; Accepted March 27, 2017

DOI: $10.3892 / \mathrm{etm} .2017 .4451$

\begin{abstract}
The feasibility of application of low-concentration contrast agent and low tube voltage combined with iterative reconstruction in whole brain computed tomography perfusion (CTP) imaging of patients with acute cerebral infarction was investigated. Fifty-nine patients who underwent whole brain CTP examination and diagnosed with acute cerebral infarction from September 2014 to March 2016 were selected. Patients were randomly divided into groups A and B. There were 28 cases in group A [tube voltage, $100 \mathrm{kV}$; contrast agent, iohexol (350 $\mathrm{mg} \mathrm{I} / \mathrm{ml})$, reconstructed by filtered back projection] and 31 cases in group B [tube voltage, $80 \mathrm{kV}$; contrast agent, iodixanol $(270 \mathrm{mg} \mathrm{I} / \mathrm{ml})$, reconstructed by algebraic reconstruction technique]. The artery $\mathrm{CT}$ value, signal-to-noise ratio (SNR), contrast-to-noise ratio (CNR), dose length product, effective dose (ED) of radiation and brain iodine intake of both groups were measured and statistically analyzed. Two physicians carried out kappa $(\kappa)$ analysis on the consistency of image quality evaluation. The difference in subjective image quality evaluation between the groups was tested by $\chi^{2}$. The differences in CT value, SNR, CNR, CTP and CT angiography subjective image quality evaluation between both groups were not statistically significant $(\mathrm{P}>0.05)$; the diagnosis rate of the acute infarcts between the two groups was not significantly different; while the ED and iodine intake in group B (dual low-dose group) were lower than group A. In conclusion, combination of low tube voltage and iterative reconstruction technique, and application of low-concentration contrast agent $(270 \mathrm{mg} \mathrm{I} / \mathrm{ml})$ in whole brain CTP examination reduced ED and iodine intake without compromising image quality, thereby reducing the risk of contrast-induced nephropathy.
\end{abstract}

Correspondence to: Dr Yibing Shi, CT Room, Xuzhou Central Hospital, 199 Jiefang Road, Xuzhou, Jiangsu 221009, P.R. China E-mail: p42599789@163.com

Key words: cerebral infarction, tomography, X-ray computer, radiation dose

\section{Introduction}

Cerebrovascular disease is one of three major diseases that can threaten human health, among which ischemic cerebrovascular disease accounts for over $80 \%$ (1). The application of 320 -slice volume computed tomography (CT) in whole brain CT perfusion (CTP) imaging can make clear diagnoses in a timely manner during the super acute period $(<6 \mathrm{~h})$ of acute cerebral infarction, evaluate blood perfusion of ischemic brain tissue, and obtain whole brain CT angiography (CTA) and CT venography (CTV) images (2). It is a method of hemodynamic evaluation widely applied clinically (3) and is important for guiding early clinical treatment, especially for thrombolytic therapy. Reducing the CT scanning radiation dose has been a focus of international studies $(4,5)$. The reduction in tube voltage can reduce the radiation dose patients receive and simultaneously reduce the amount of contrast agent needed to make it more suitable for brain perfusion imaging (6). The present study was designed to investigate the application feasibility of the dual low-dose scanning scheme in cerebral infarction whole brain CTP by comparing whole brain CTP image quality and radiation dose of patients with acute cerebral infarction under different tube voltages and contrast agent concentration scanning schemes.

\section{Patients and methods}

Patients. Fifty-nine patients who underwent 320-slice whole brain CTP and diagnosed with acute cerebral infarction in Xuzhou Clinical Medical College from September 2014 to March 2016 were enrolled (excluding those with allergic history to iodinated contrast agent, renal insufficiency and those taking metformin for thyroid disease). The patients included 35 males and 24 females, aged 39-86 years, with mean age of $60.0 \pm 14.6$ years. The height and weight of all patients were measured before examination and body mass index (BMI) was calculated. Patients were randomly divided into groups A and B and the general parameters are shown in Table I. The present study was approved by the Ethics Committee of Xuzhou Central Hospital and written informed consent was obtained from all the patients.

Examination methods. An Aquilion 320 volume CT (Toshiba, Ohtawara, Japan) was used for whole brain CTP 
Table I. Basic parameters of patients in groups A and B.

\begin{tabular}{lccc}
\hline Groups & $\begin{array}{c}\text { Case no. } \\
(\text { male/female })\end{array}$ & $\begin{array}{c}\text { Age } \\
(\text { years })\end{array}$ & $\begin{array}{c}\mathrm{BMI} \\
\left(\mathrm{kg} / \mathrm{m}^{2}\right)\end{array}$ \\
\hline Group A & $28(18 / 10)$ & $61.8 \pm 11.7$ & $24.2 \pm 3.6$ \\
Group B & $31(17 / 14)$ & $58.3 \pm 16.8$ & $24.2 \pm 3.3$ \\
P-value & 0.648 & 0.942 & 0.984 \\
\hline
\end{tabular}

BMI, body mass index.

scanning. For group A, the tube voltage was $100 \mathrm{kV}$ and the contrast agent was iohexol $(350 \mathrm{mg} \mathrm{I} / \mathrm{ml})$. For group B, the tube voltage was $80 \mathrm{kV}$ and the contrast agent was iodixanol ( $270 \mathrm{mg} \mathrm{I} / \mathrm{ml}$ ). The other scanning parameters in both groups were the same: The volume scanning mode was used, the thickness was $0.5 \mathrm{~mm}$ and the tube current was $310 \mathrm{~mA}$. The proportional total injection volume $(50 \mathrm{ml})$ and flow rate $(4 \mathrm{ml} / \mathrm{sec})$ in both groups were the same. The whole brain perfusion scanning scheme was used and the first exposure began $7 \mathrm{sec}$ after contrast agent injection. The exposure time interval was $2 \mathrm{sec}$ during the arterial phase, $4-6$ sec during the venous phase, and total scanning time was $62 \mathrm{sec}$. A total of 19 exposures were achieved at 7, 11, 13, 15, 19, 21, 23, 25, 27, $29,32,34,36,38,42,47,52,57$ and $62 \mathrm{sec}$ after injection of contrast agent (Table II).

Reconstruction method. Images from group A were reconstructed with the conventional filtered back projection (FBP) technique and group B with the algebraic reconstruction technique (ART).

Post-processing of images. A total of 6,080 images divided across 19 groups obtained from scanning were imported to the Toshiba workstation (Toshiba, Tokyo, Japan) and entered into the 4-D perfusion software for analysis (version 3.2; Toshiba). The region of interest (ROI) was selected for CT value measurement in the arterial M1 segment of the brain that the software was used to automatically generate, including other perfusion parameters. CTP referred to repeating multiple scans on the same selected layer continuously during intravenous injection of contrast agent to obtain time-density curves (TDC) (which reflected changes in contrast agent concentrations in organs, and indirectly reflected changes in organ perfusion volume) per pixel on this layer. Cerebral blood flow $(\mathrm{CBF})$, relative cerebral blood volume (rCBV), mean transit time (MTT) and time to peak (TTP) were calculated with different mathematical models based on the curve. These parameters were used to form a new digital matrix. Intuitional and clear parameter color images were obtained through digital-to-analog conversion and pseudo-color processing, named CTP. The Toshiba Aquilion One 320 volume CT (Toshiba) was equipped with a $160-\mathrm{mm}$ range detector (Barui Medical Equipment Co., Beijing, China), which could obtain images of the whole brain under a single exposure, thus whole brain perfusion scanning can be achieved without moving the scanning table. In addition, it can achieve whole brain isotropy, and obtain whole brain horizontal, coronal and sagittal CTP images. Finally, brain CTA and CTV images were obtained through post-processing at the workstation.

All volume data were imported to the Toshiba Vital version 6.5.3 workstation (Toshiba) and whole brain CTA and CTV images were obtained with the digital silhouette technology of the workstation.

Image quality evaluation. i) Objective evaluation. CT value at the arterial M1 segment of the brain at the peak in the images of groups A and B were measured and the signal-to-noise ratio (SNR) and contrast-to-noise ratio (CNR) were calculated based on formula (1) and formula (2): (1) SNR = ROI mean CT value/image noise; $(2) \mathrm{CNR}=(\mathrm{ROI}$ mean $\mathrm{CT}$ value - mean $\mathrm{CT}$ value at the same layer of brain parenchyma)/image noise.

Image noise $=$ standard deviation of the measurement value by $\mathrm{CT}$ within the ROI range. Calcification should be avoided to the greatest extent in ROI selections and its area should be over one-half of the lumen area (Fig. 1A and B).

ii) Subjective evaluation. Two experienced radiologists independently completed the image quality rating without knowledge of the groupings, and they adopted a 3-level scoring system: A score of 3 indicated excellent image quality with obvious pseudo-color difference, the abnormal perfusion area could be easily identified and the diagnosis was clear; a score of 2 indicated general image quality, relatively obvious pseudo-color difference and the abnormal perfusion area could be identified by adjusting the contrast; a score of 1 indicated poor image quality, blurred pseudo-color difference

Table II. Scanning schemes in groups A and B.

\begin{tabular}{|c|c|c|c|c|c|c|c|c|}
\hline \multirow{2}{*}{$\begin{array}{l}\text { Scanning } \\
\text { sequence } \\
\text { (exposure } \\
\text { frequency) }\end{array}$} & \multicolumn{2}{|c|}{$\begin{array}{l}\text { Tube voltage } \\
\qquad(\mathrm{kV})\end{array}$} & \multicolumn{2}{|c|}{$\begin{array}{l}\text { Tube current } \\
\qquad(\mathrm{mA})\end{array}$} & \multicolumn{2}{|c|}{$\begin{array}{l}\text { Exposure time (sec) } \\
\text { (time after injection } \\
\text { of contrast agent) }\end{array}$} & \multicolumn{2}{|c|}{$\begin{array}{l}\text { Exposure time } \\
\text { interval }(\mathrm{sec})\end{array}$} \\
\hline & Group A & Group B & Group A & Group B & Group A & Group B & Group A & Group B \\
\hline $1(1)$ & 100 & 80 & 310 & 310 & 7 & 7 & - & - \\
\hline $2(3)$ & 100 & 80 & 150 & 150 & $11-15$ & $11-15$ & 2 & 2 \\
\hline $3(6)$ & 100 & 80 & 310 & 310 & $19-29$ & $19-29$ & 2 & 2 \\
\hline $4(4)$ & 100 & 80 & 150 & 150 & $32-38$ & $32-38$ & 2 & 2 \\
\hline $5(5)$ & 100 & 80 & 310 & 310 & $47-62$ & $47-62$ & 5 & 5 \\
\hline
\end{tabular}



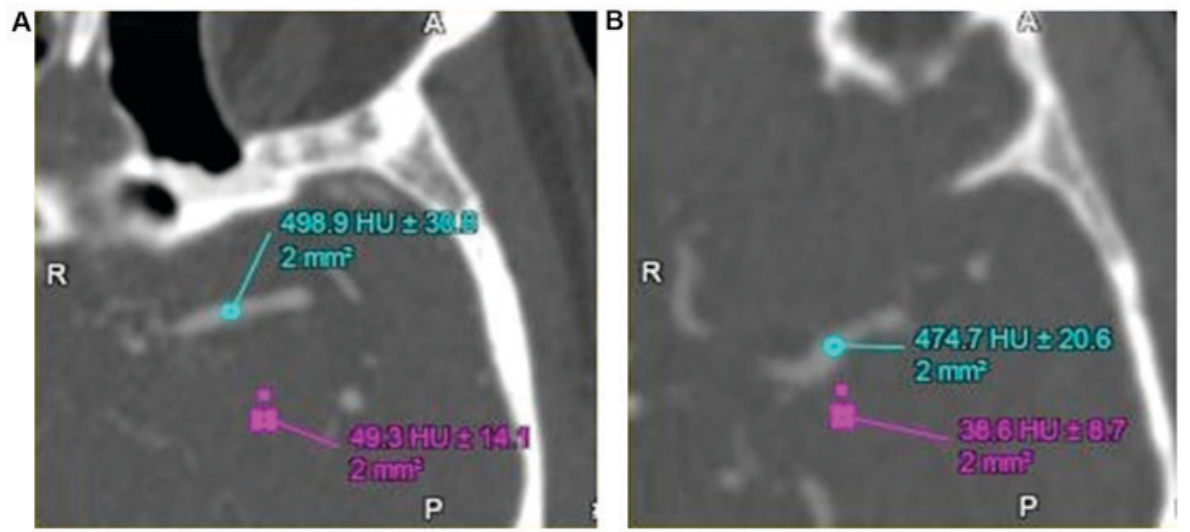

Figure 1. The objective evaluation of the image quality. (A) A 71-year-old male presented with sudden dizziness for no obvious cause $2 \mathrm{~h}$ before evaluation, accompanied with salivation. The figure shows the horizontal axis surface at the arterial M1 segment of the brain of a representative patient in group A. Tube voltage, $100 \mathrm{kV}$; contrast agent, iohexol $(350 \mathrm{mg} \mathrm{I} / \mathrm{ml})$, reconstructed with FBP. An area of $2 \mathrm{~mm}^{2}$ was selected as the ROI, and the CT value at the arterial M1 segment in the left brain was measured as $498.9 \mathrm{HU} \pm 30.8$ and the $\mathrm{CT}$ value of the brain parenchyma on the same layer was $49.3 \mathrm{HU} \pm 30.8$. (B) A 59-year-old male presented with sudden double vision, accompanied with dizziness and headache. The figure shows the horizontal axis surface at the arterial M1 segment of the brain of a representative patient in group B. Tube voltage, $80 \mathrm{kV}$; contrast agent, iodixanol $(270 \mathrm{mg} \mathrm{I} / \mathrm{ml})$, reconstructed with algebraic reconstruction technique. An area of $2 \mathrm{~mm}^{2}$ was selected as the ROI, and the CT value at the arterial M1 segment in the left brain was measured as $474.7 \mathrm{HU} \pm 20.6$ and the CT value of the brain parenchyma on the same layer was $38.6 \mathrm{HU} \pm 8.7$. FBP, filtered back projection; ROI, region of interest; CT, computed tomography.

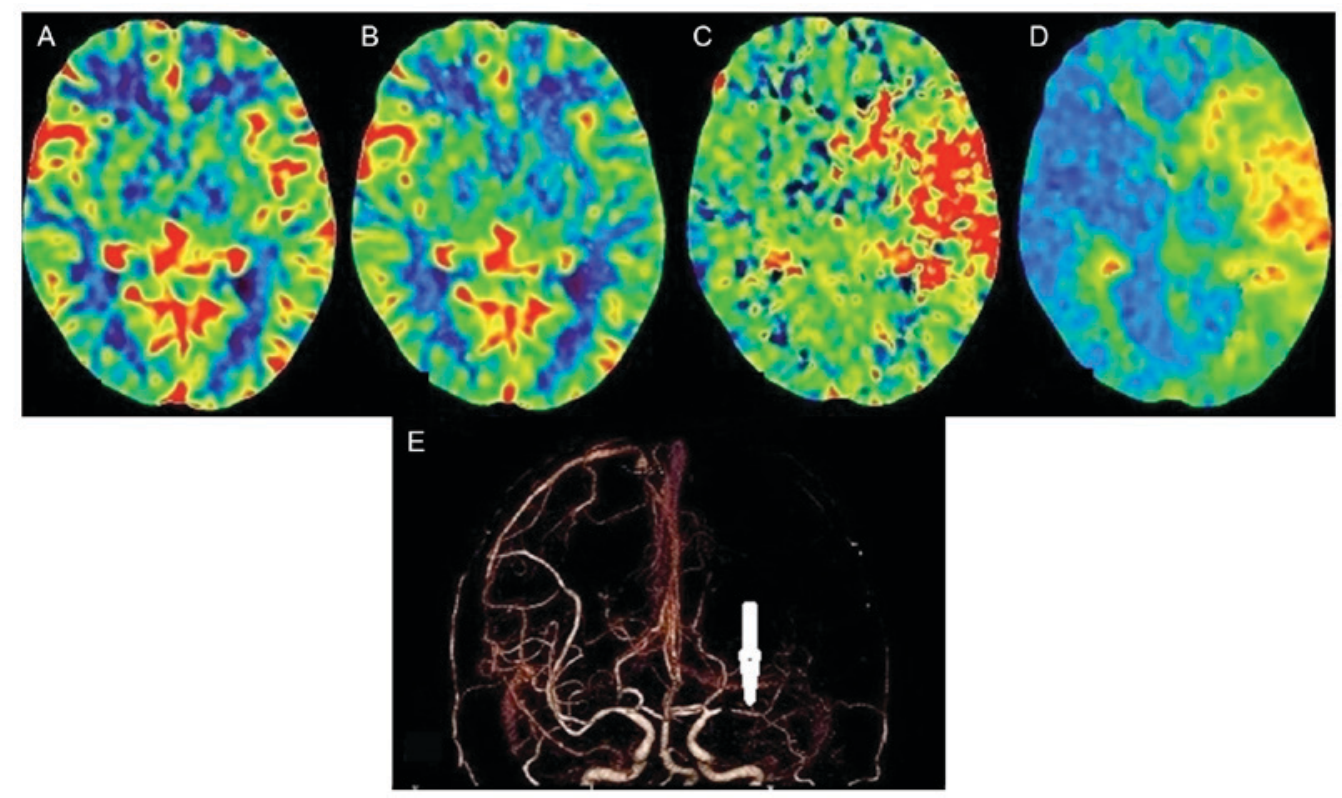

Figure 2. A 55-year-old male presented with slurred speech, accompanied with right limb weakness for $3 \mathrm{~h}$. The scanning scheme in group A (100 kV, $350 \mathrm{mg}$ $\mathrm{I} / \mathrm{ml}$ iohexol, reconstructed by FBP) was used. The subjective image quality score by two physicians was 3 ; the clinical symptoms were positive, CTP and CTA were consistent with the clinical symptoms, the matching rate score of the clinical symptoms and imaging diagnosis rate was 2 . (A) The cerebral perfusion CBV image shows that the CBV of the frontotemporal parietal lobes on both sides were basically symmetrical. (B) The cerebral perfusion CBF image shows that CBF of the frontotemporal parietal lobes on both sides were substantially symmetrical. (C) The cerebral perfusion MTT image shows the left frontotemporal lobe MTT was extended (compared with the opposite side, there was a large area of red time extension on the left frontotemporal lobe and the time was short to the extent of long pseudo-color change: Blue $\rightarrow$ green $\rightarrow$ yellow $\rightarrow$ red). (D) The cerebral perfusion TTP image shows the left frontotemporal lobe TTP was extended (there was a large area of red and yellow time extension area on the left frontotemporal lobe). (E) CTA image shows there was a distal occlusion (arrow) at arterial M1 segment of the left brain. FBP, filtered back projection; CTP, computed tomography perfusion; CTA, CT angiography; MTT, mean transit time.

and the abnormal perfusion area or split layer in the image could not be identified after contrast adjustment. CTP images could not be generated and diagnostic needs were not met (7). The consistency of image quality evaluated by the two physicians was assessed by kappa $(\kappa)$ analysis. $\kappa$ value, 0.8-1.0 indicated the consistency was excellent; 0.61-0.8 indicated the consistency was better; $0.41-0.6$ indicated the consistency was ordinary; and $<0.4$ indicated poor consistency.
The matching degree between the abnormal area shown in the images and the symptoms of patients were evaluated, combined with the clinical symptoms of patients according to whole brain CTA and CTP images. The matching degree was evaluated with a 0-2 level scoring system: A score of 2 indicated the CTP hypoperfusion area occurred in the responsible lesion (CBV and CBF were decreased, MTT and TTP were extended), and stenosis or occlusion occurred in the 


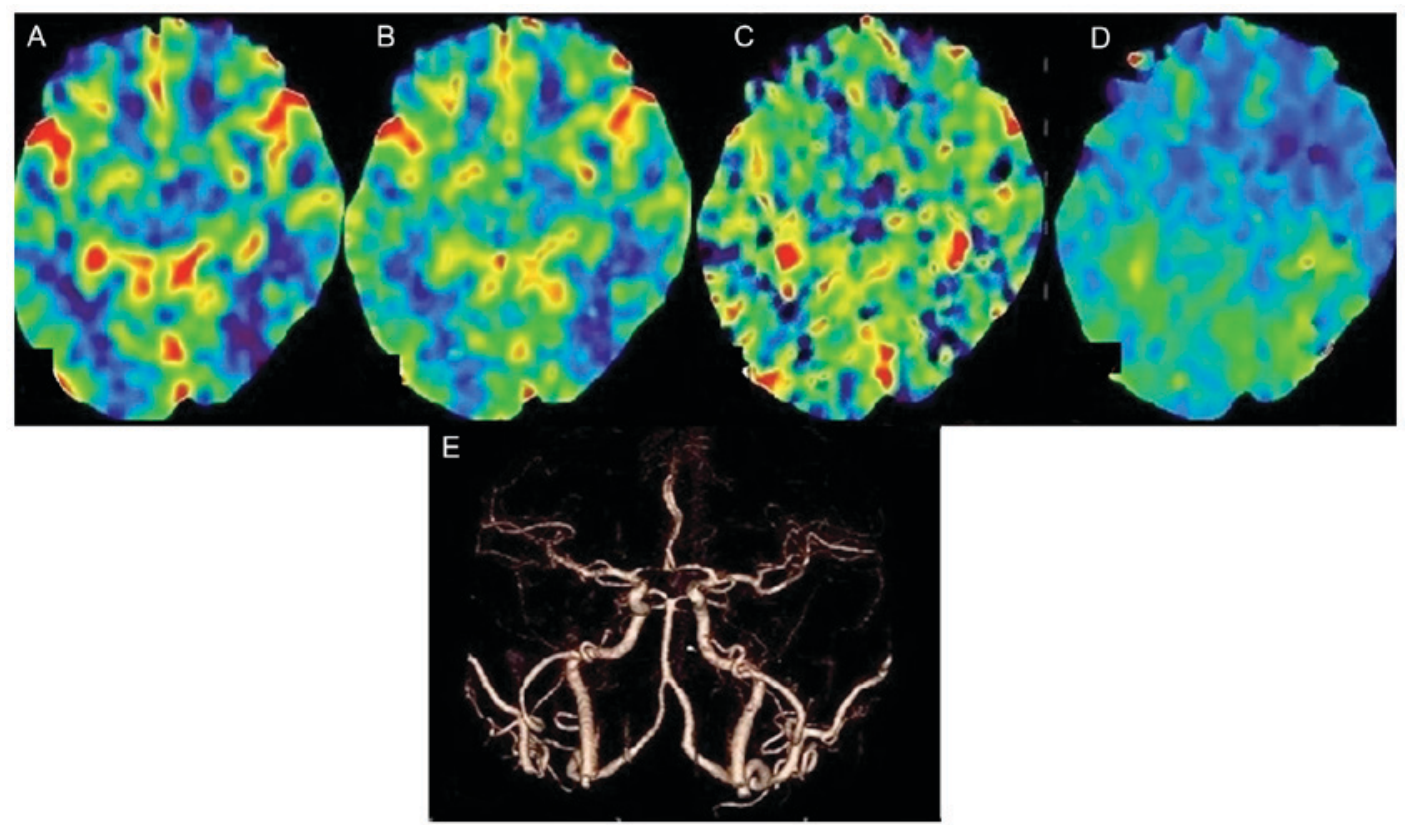

Figure 3. A 50-year-old male, presented with dizziness, accompanied with nausea for no obvious cause $2.5 \mathrm{~h}$ before examination. Group B ( $80 \mathrm{kV}, 270 \mathrm{mg} \mathrm{I} / \mathrm{ml}$ iodixanol, reconstructed by ART). The subjective image quality score by the two physicians was 2 ; the clinical symptoms were positive, CTP was positive, CTA was negative and the matching degree score of the clinical symptoms and imaging diagnosis was 1. (A) The CBV image shows the CBVs of the frontotemporal parietal lobe on both sides were basically symmetrical. (B) The CBF image shows the CBFs of the frontotemporal parietal lobe on both sides were substantially symmetrical. (C) The cerebral perfusion MTT image shows the MTT of the frontotemporal lobes on both side was substantially symmetrical (D) The cerebral perfusion transit TTP image shows the right frontotemporal lobe TTP was extended compared with the left (there was partial green time extension area on the right frontotemporal lobe relative to the left original blue zone). (E) CTA image shows there was no significant arterial stenosis in the brain. ART, algebraic reconstruction technique; CTP, computed tomography perfusion; CTA, CT angiography; CBV, cerebral blood volume; CBF, cerebral blood flow; MTT, mean transit time; TTP, time to peak.

Table III. Comparison of CT value, SNR and CNR at arterial M1 segment of the brain between groups A and B (mean \pm SD).

\begin{tabular}{|c|c|c|c|c|c|c|c|c|c|}
\hline \multirow[b]{2}{*}{ Indicator } & \multicolumn{3}{|c|}{ Total value } & \multicolumn{3}{|c|}{ BMI $<23 \mathrm{~kg} / \mathrm{m}^{2}$ subgroup } & \multicolumn{3}{|c|}{ BMI $\geq 23 \mathrm{~kg} / \mathrm{m}^{2}$ subgroup } \\
\hline & Group A & Group B & P-value & Group A & Group B & P-value & Group A & Group B & P-value \\
\hline $\begin{array}{l}\text { CT value } \\
\text { (HU) }\end{array}$ & $495.7 \pm 125.4$ & $496.0 \pm 125.2$ & 0.993 & $548.5 \pm 125.6$ & $512.9 \pm 145.2$ & 0.550 & $466.4 \pm 118.6$ & $485.3 \pm 113.6$ & 0.623 \\
\hline SNR & $20.9 \pm 11.5$ & $17.9 \pm 7.8$ & 0.234 & $21.3 \pm 10.2$ & $16.3 \pm 6.8$ & 0.178 & $20.7 \pm 12.4$ & $18.9 \pm 8.3$ & 0.609 \\
\hline CNR & $18.7 \pm 10.3$ & $15.8 \pm 7.0$ & 0.209 & $19.4 \pm 4.5$ & $14.4 \pm 6.2$ & 0.156 & $18.4 \pm 10.9$ & $16.7 \pm 7.6$ & 0.600 \\
\hline
\end{tabular}

CT, computed tomography; SNR, signal-to-noise ratio; CNR, contrast-to-noise ratio; BMI, body mass index.

corresponding artery; a score of 1 indicated CTP hypoperfusion occurred in the responsible lesion (CBV and $\mathrm{CBF}$ were decreased, MTT and TTP were extended), but no obvious stenosis or occlusion occurred in the corresponding artery, or stenosis of the responsible artery occurred but a CTP low perfusion area was not shown; a score of 0 indicated the symptoms of patients were distinct but CTP and CTA were negative (8) (Figs. 2 and 3).

Effective dose (ED) of radiation and iodine intake. The dose length product (DLP) of all patients was recorded and the ED values were calculated according to formula [3]. [3] ED = DLP x k; k, conversion factor; in the present study, $\mathrm{k}=0.0021 \mathrm{mSv} /(\mathrm{mGy} \cdot \mathrm{cm})$. Formula [4] was used to calculate the iodine intakes of the patients in both groups. [4] Iodine intake $=$ iodine concentration $\mathrm{x}$ contrast agent dose.
The display rate of infarction lesions. The number of patients diagnosed with acute cerebral infarctions were recorded in the two groups and the infarct display rates were compared.

The adverse reactions of contrast agents. Patients were asked about their comfort within 15-20 min after scanning. Simultaneously, a variety of adverse reactions were recorded within 20 min after scanning and within 3 days during the follow-up period.

Statistical analysis. SPSS 16.0 statistical software (SPSS, Inc., Chicago, IL, USA) was used for data analysis. Measurement data are expressed as mean $\pm \mathrm{SD}$. Comparisons of measurement data between groups were by t-test. Comparisons of subjective image scores and qualitative data were by $\chi^{2}$ test. $\mathrm{P}<0.05$ was considered to indicate a statistically significant difference. 
Table IV. CTP image quality scores and comparison between groups A and B by two physicians.

\begin{tabular}{|c|c|c|c|c|c|c|c|c|}
\hline \multirow[b]{2}{*}{ Score } & \multicolumn{3}{|c|}{ Group A } & \multicolumn{3}{|c|}{ Group B } & \multirow[b]{2}{*}{$\chi^{2}$} & \multirow[b]{2}{*}{ P-value } \\
\hline & 1 & 2 & 3 & 1 & 2 & 3 & & \\
\hline Physician 1 & 1 & 11 & 16 & 0 & 7 & 24 & 3.345 & 0.188 \\
\hline Physician 2 & 1 & 10 & 17 & 0 & 10 & 21 & 1.272 & 0.529 \\
\hline
\end{tabular}

Group A, $\kappa=0.651, \mathrm{P}<0.001$; group $\mathrm{B}, \kappa=0.760, \mathrm{P}<0.001$. CTP, computed tomography perfusion.

Table V. Matching degree score and comparison of the image display and symptoms between groups $\mathrm{A}$ and $\mathrm{B}$.

\begin{tabular}{lccc}
\hline Groups & Score 2 & Score 1 & Score 0 \\
\hline Group A & 11 & 6 & 11 \\
Group B & 13 & 9 & 9 \\
\hline
\end{tabular}

$\chi^{2}=0.816, \mathrm{P}=0.665$.

Table VI. Comparison of indicators between groups A and B.

\begin{tabular}{lcc}
\hline Indicator & Group A & Group B \\
\hline Tube voltage $(\mathrm{kV})$ & 100 & 80 \\
DLP $(\mathrm{mGy} \cdot \mathrm{cm})$ & $5,743.50$ & $3,131.10$ \\
ED $(\mathrm{mSv})$ & 12.06 & 6.58 \\
Contrast agent dose $(\mathrm{ml})$ & 50 & 50 \\
Iodine intake $(\mathrm{mg})$ & 17,500 & 13,500 \\
\hline
\end{tabular}

DLP, dose length product; ED, effective dose.

\section{Results}

The general parameters and comparisons of the 59 patients are shown in Table I. The differences in age, gender and BMI between the two groups were not statistically significant $(\mathrm{P}>0.05)$.

The comparison of the CT value, SNR and CNR at the peak of the arterial M1 segment in the brain between groups $A$ and B is shown in Table III. The differences in CT value, SNR and CNR between the two groups were not statistically significant $(\mathrm{P}>0.05)$.

The evaluations of image quality of groups A and B by the two physicians were consistent (group $\mathrm{A}, \kappa$ value was $0.651, \mathrm{P}<0.001$; group $\mathrm{B}, \kappa$ value was $0.760, \mathrm{P}<0.001)$. The differences in image quality between groups $\mathrm{A}$ and $\mathrm{B}$ were not statistically significant (physician $1, \chi^{2}=3.345, \mathrm{P}>0.05$; physician $2, \chi^{2}=1.272, \mathrm{P}>0.05$; Table IV). In the evaluation of matching degree of image display and symptoms, the difference between groups A and B was not statistically significant ( $\mathrm{P}>0.05$ ) (Table V and Figs. 2 and 3).

DLP, ED, contrast agent dose and iodine intake in groups A and $\mathrm{B}$ are shown in Table VI. The ED decreased by $5.48 \mathrm{mSv}$ $(45 \%)$ in group B compared with group A. Iodine intake in
Table VII. Comparison of diagnosis rate of acute infarct between groups A and B.

\begin{tabular}{llllllllll}
\hline & \multicolumn{4}{c}{ Group A } & & & \multicolumn{3}{c}{ Group B } \\
\cline { 2 - 4 } \cline { 5 - 8 } Scores & 0 & 1 & 2 & 3 & & 0 & 1 & 2 & 3 \\
\hline No. of lesions & 8 & 15 & 5 & 0 & 9 & 18 & 3 & 1 \\
\hline
\end{tabular}

$\chi^{2}=1.683, \mathrm{P}=0.641$.

group B was reduced by 4,000 $\mathrm{mg}$ (23\%) compared with groups A (Table VI).

Among 28 patients in group A, there were eight without acute infarct, 15 with one infarct, and five with two infarcts. Among 31 patients in group $\mathrm{B}$, there were nine diagnosed without acute infarct, 18 with one infarct, three with two infarcts and one with three infarcts. The results showed that the rate of diagnosis of infarcts was not statistically significant between groups A and B $\left(\chi^{2}=1.683, \mathrm{P}>0.05\right)$ (Table VII).

The 59 patients were without extravasation of contrast agent during scanning. There were no significant allergic reactions within 15-20 min after scanning. Within 3 days during the follow-up period, four patients had moderate allergic reactions [two patients in group A $(2 / 28,7.1 \%)$, two patients in group B (2/31, 6.5\%)]. Two patients manifested with facial rash and swelling (one patients in each group), one patient manifested abdominal urticaria (group A) and one with vomiting (group B).

\section{Discussion}

The 320-slice whole brain CTP is a form of 'one-stop' CT examination for hyperacute cerebral infarction that includes the joint application of routine CTP, CTA and CTV. Some refer to it as a multi-mode CT examination (9). However, because multiple perfusion images require continuous exposure, subjects will suffer from higher radiation doses. In recent years, reducing the radiation dose has been a focus of international research (10-12). Recent studies (13-15) show that low $\mathrm{kV}$ scanning can cause average $\mathrm{X}$-ray photon energy closer to the iodine K-edge and decrease Compton scattering, so that CT values will be increased, while radiation doses will be reduced.

With increasing application of contrast agents, the incidence of contrast-induced nephropathy (CIN) has significantly increased. According to a previous study, in addition to the osmotic pressure factor, the viscosity of contrast agents are a 
key factor in determining the incidence of CIN (16). Therefore, it is worth investigating how the isotonic contrast agent, iodixanol can be modified by reducing its viscosity to make it closer to physiological characteristics, while maintaining its isotonic characteristics. According to the isotonic iodine effect, the dose of contrast agent must be increased to make $270 \mathrm{mg} \mathrm{I} / \mathrm{ml}$ iodixanol reach the development effect of $350 \mathrm{mg}$ $\mathrm{I} / \mathrm{ml}$ iohexol, and the latter increase will lead to increased risk of CIN (17). In the present study, it was shown that the low $\mathrm{kV}$ scheme (group B) X-ray output energy was closer to the $33 \mathrm{keV}$ iodine $\mathrm{K}$-edge, and no decrease in intracranial $\mathrm{CT}$ value was observed by comparing different $\mathrm{kV}$ scanning schemes between the two groups.

With the progress of technology, ART is increasingly applied in the CT reconstruction scheme. The latest ART reconstruction can not only effectively reduce the image noise and improve image SNR and CNR, thus maintaining or even improving the image quality, but can also make up for the crease in image noise and decrease in image quality from low $\mathrm{kV}$, thereby reducing the $\mathrm{ED}(17)$.

The results of the present study showed that the differences in CT value, SNR, CNR, CTP and CTA subjective image quality evaluations between group B (iodixanol, $270 \mathrm{mg} \mathrm{I} / \mathrm{ml}$ ) and $80 \mathrm{kV}$ scanning scheme combined with ART reconstruction) and group A (iohexol, $350 \mathrm{mg} \mathrm{I} / \mathrm{ml}$ ) and $100 \mathrm{kV}$ scanning scheme combined with FBP reconstruction) were not statistically significant $(P>0.05)$. All imaging modalities met the needs of clinical diagnosis; there was no significant difference in the capacity of finding acute infarcts between the groups $(\mathrm{P}>0.05)$; the $\mathrm{ED}$ and iodine intake in group $\mathrm{B}$ (dual low-dose group) were significantly lower than in group A. Therefore, the application of dual low-dose scanning scheme combined with iterative reconstruction techniques can reduce ED suffered by patients, reduce intake of iodine, meet the needs of clinical diagnosis and minimize possible side effects.

The limitations of the present study were: i) The partial artery lumens in the brain were small, ROI selection generated artifacts easily because of the partial volume effect; ii) the sample size was small. Large sample sizes are required for further confirmation; and iii) in evaluating patients with intracranial arterial stenosis, the comparison was not made with the gold standard (DSA) control, therefore its diagnostic accuracy of stenosis could not be evaluated.

\section{References}

1. Jeng JS and Yip PK: Epidemiology of cerebrovascular disease inthe elderly in East Asia. Geriatr Gerontol Int 4: S198-S201, 2004.

2. Fu J, Chen WJ, Wu GY, Cheng JL, Wang MH, Zhuge Q, Li JC, Zhang Q, Zhang Y, Xia NZ, et al: Whole-brain 320-detector row dynamic volume $\mathrm{CT}$ perfusion detected crossed cerebellar diaschisis after spontaneous intracerebral hemorrhage. Neuroradiology 57: 179-187, 2015.

3. National Lung Screening Trial Research Team; Aberle DR, Adams AM, Berg CD, Black WC, Clapp JD, Fagerstrom RM Gareen IF, Gatsonis C, Marcus PM and Sicks JD: Reduced lung-cancer mortality with low-dose computed tomographic screening. N Eng J Med 365: 395-409, 2011.
4. Kulkarni NM, Uppot RN, Eisner BH and Sahani DV: Radiation dose reduction at multidetector $\mathrm{CT}$ with adaptive statistical iterative reconstruction for evaluation of urolithiasis: how low can we go? Radiology 265: 158-166, 2012.

5. Halliburton SS, Abbara S, Chen MY, Gentry R, Mahesh M, Raff GL, Shaw LJ and Hausleiter J; Society of Cardiovascular Computed Tomography: SCCT guidelines on radiation dose and dose-optimization strategies in cardiovascular CT. J Cardiovasc Comput Tomogr 5: 198-224, 2011.

6. Nakaura T, Nakamura S, Maruyama N, Funama Y, Awai K, Harada K, Uemura S and Yamashita Y: Low contrast agent and radiation dose protocol for hepatic dynamic CT of thin adults at 256-detector row CT: effect of low tube voltage and hybrid iterative reconstruction algorithm on image quality. Radiology 264: 445-454, 2012.

7. Chen GZ, Zhang LJ, Schoepf UJ, Wichmann JL, Milliken CM, Zhou CS, Qi L, Luo S and Lu GM: Radiation dose and image quality of $70 \mathrm{kVp}$ cerebral CT angiography with optimized sinogram-affirmed iterative reconstruction: comparison with $120 \mathrm{kVp}$ cerebral CT angiography. Eur Radiol 25: 1453-1463, 2015.

8. Escudero D, Otero J, Marqués L, Parra D, Gonzalo JA, Albaiceta GM, Cofiño L, Blanco A, Vega P, Murias E, et al: Diagnosing brain death by $\mathrm{CT}$ perfusion and multislice $\mathrm{CT}$ angiography. Neurocrit Care 11: 261-271, 2009.

9. Nakayama Y1, Awai K, Funama Y, Liu D, Nakaura T, Tamura Y and Yamashita Y: Lower tube voltage reduces contrast material and radiation doses on 16-MDCT aortography. AJR Am J Roentgenol 187: 490-497, 2006.

10. Marin D, Nelson RC, Schindera ST, Richard S, Youngblood RS, Yoshizumi TT and Samei E: Low-tube-voltage, high-tube-current multidetector abdominal CT: improved image quality and decreased radiation dose with adaptive statistical iterative reconstruction algorithm - initial clinical experience. Radiology 254: 145-153, 2010.

11. Heyer CM, Mohr PS, Lemburg SP, Peters SA and Nicolas V: Image quality and radiation exposure at pulmonary $\mathrm{CT}$ angiography with 100 - or $120-\mathrm{kVp}$ protocol: prospective randomized study. Radiology 245: 577-583, 2007.

12. Ketelsen D, Horger M, Buchgeister M, Fenchel M, Thomas C, Boehringer N, Schulze M, Tsiflikas I, Claussen CD and Heuschmid M: Estimation of radiation exposure of 128-slice 4D-perfusion CT for the assessment of tumor vascularity. Korean J Radiol 11: 547-552, 2010.

13. Tomizawa N, Nojo T, Akahane M, Torigoe R, Kiryu S and Ohtomo K: AdaptiveIterative dose reduction in coronary CT angiography using 320-row CT: assessment of radiation dose reduction and image quality. J Cardiovasc Comput Tomogr 6: 318-324, 2012.

14. Wang R, Schoepf UJ, Wu R, Reddy RP, Zhang C, Yu W, Liu Y and Zhang Z: Image quality and radiation dose of low dose coronary CT angiography in obese patients: sinogram affirmed iterative reconstruction versus filtered back projection. Eur J Radiol 81: 3141-3145, 2012.

15. Hirata M, Sugawara Y, Fukutomi Y, Oomoto K, Murase K, Miki $\mathrm{H}$ and Mochizuki T: Measurement of radiation dose in cerebral CT perfusion study. Radiat Med 23: 97-103, 2005.

16. Dittrich R, Akdeniz S, Kloska SP, Fischer T, Ritter MA, Seidensticker P, Heindel W, Ringelstein EB and Nabavi DG: Low rate of contrast-induced nephropathy after CT perfusion and CT angiography in acute stroke patients. J Neurol 254: 1491-1497, 2007.

17. Zheng M, Liu Y, Wei M, Wu Y, Zhao H and Li J: Low concentration contrast medium for dual-source computed tomography coronary angiography by a combination of iterative reconstruction and low-tube-voltage technique: feasibility study. Eur J Radiol 83: e92-e99, 2014. 